\title{
Reversal of Daunorubicin Resistance in P388/ADR Cells by Itraconazole
}

Sudhir Gupta, Jungmee Kim, and Sastry Gollapudi

Division of Basic and Clinical Immunology, University of California, Irvine, California 92717

\begin{abstract}
Itraconazole is a recently developed triazole antifungal agent that inhibits cell membrane sterol biosynthesis. Itraconazole, in a dose-dependent manner, enhanced intracellular accumulation of daunorubicin and reversed the drug resistance in murine leukemia P388/ADR cells. In addition, itraconazole corrected the altered plasma membrane potentials of P388/ADR cells. The concentrations of itraconazole that reversed drug resistance are comparable to the plasma levels achieved by therapeutic dosage used in the treatment of fungal infections. Therefore, itraconazole is a potential candidate for in vivo use to reverse multidrug resistance in cancer with added benefit of its antifungal property. (J. Clin. Invest. 1991.87:1467-1469.) Key words: P-glycoprotein - multidrug resistance • plasma membrane potential $\bullet$ drug accumulation $\bullet$ triazoles
\end{abstract}

\section{Introduction}

Multidrug resistance (MDR) ${ }^{1}$ remains the major obstacle in the therapeutic cure of many malignancies. A number of mechanisms for multidrug resistance at the level of plasma membrane, cytosol, and the DNA levels, have been suggested (1-3). The most common mechanism in the majority of MDR cell lines is an over expression of P-glycoprotein, a 170-kD metabolically active efflux pump glycoprotein (4). A number of calcium channel blockers and camodulin inhibitors have been shown to reverse multidrug resistance both in vitro and in vivo $(3,5)$. Recently we have shown that cyclosporin A, a lipophilic agent that partitions between plasma membranes, reverses multidrug resistance both in vitro and in vivo $(5,6)$. Itraconazole, a new generation 1,2,4 triazole antifungal agent that inhibits different cytochrome P-450 systems (7-9), has been shown to synergize with cyclosporin A (10). Therefore, we set out to explore the possibility of whether similar synergism would exist between these two compounds in reversing MDR in vitro. As a part of the control experiment before doing experiments of synergism, we discovered that itraconazole alone

Received for publication 25 October 1990 and in revised form $10 \mathrm{De}$ cember 1990.

1. Abbreviations used in this paper: CsA, cyclosporin A; DiOC5, 3-3 dipentyloxacarbocyanine iodide; DNR, daunorubicin; MDR, multidrug resistance.

J. Clin. Invest.

(c) The American Society for Clinical Investigation, Inc.

$0021-9738 / 91 / 04 / 1467 / 03 \quad \$ 2.00$

Volume 87, April 1991, 1467-1469 corrects intracellular drug accumulation and altered plasma membrane potentials and reverses drug resistance in murine P388/ADR cells in vitro.

\section{Methods}

Cell lines. P388 (parental sensitive) and P388/ADR (MDR) cell lines were a gift from Dr. Lewis Slater of the University of California, Irvine. These cell lines are maintained in tissue culture in medium RPMI- 1640 supplemented with $10 \%$ FCS. P388/ADR is primarily resistant to adriamycin and cross-resistant to vincristine and daunorubicin. An over expression of P-glycoprotein appears to be the major mechanism of MDR in this cell line.

Chemicals. Daunorubicin was purchased from Sigma Chemical Co., St. Louis, MO. ${ }^{3} \mathrm{H}$ thymidine was purchased from ICN Pharmaceuticals, Costa Mesa, CA. Itraconazole (R 51211), \pm cis-4-[4[4-[4-2-(2,4 dichlorophenyl)-2-(1-H-1,2,4-triazol-1-ylmethyl)-1,3dioxolan - $4-y l$ ]methoxy]phenyl]-1-piperazinyl]phenyl]-2,4-dihydro2-(1-methylpropyl)-3-H-1,2,4-triazol-3-one, was a gift from Janssen Pharmaceutical, Beerse, Belgium. Itraconazole was dissolved in dimethylefloride (DMF). DMF alone was used as a control in all the experiments. Carbocyanine dye (DiOC5) was purchased from Molecular Probes, Inc., Eugene, OR.

Daunorubicin accumulation. $1 \times 10^{6} \mathrm{cells} / \mathrm{ml}$ were resuspended in $12 \times 75-\mathrm{mm}$ culture tubes in the presence or absence of various concentrations of itraconazole. Daunorubicin $(4 \mu \mathrm{g} / \mathrm{ml})$ was added to the cells, gently mixed, and incubated at $37^{\circ} \mathrm{C}$. Tubes were removed at 45 min and cells for intracellular daunorubicin accumulation were analyzed by FACScan (Becton-Dickinson Co., San Jose, CA). 10,000 cells were counted. All experiments were done in triplicate. Mean fluorescence is recorded from the histogram and data are expressed as mean fluorescence channel numbers.

Drug sensitivity. To determine the reversibility of drug resistance, we incubated cells with various concentrations of daunorubicin in the presence or absence of various concentrations of itraconazole for $1 \mathrm{~h}$ at $37^{\circ} \mathrm{C}$. Cells were pulsed with ${ }^{3} \mathrm{H}$ thymidine, harvested, and ${ }^{3} \mathrm{H}$ thymidine incorporation was measured, using scintillation counter. Data are expressed as IC/50 (molar concentration of daunorubicin required for $50 \%$ inhibition of DNA synthesis).

Plasma membrane potentials. Plasma membrane potentials were measured with carbocyanine dye DiOC5, using FACScan by a modification of a previously described technique (11). In brief, cells are washed with PBS and resuspended in PBS supplemented with 5\% PBS. $1 \times 10^{6} \mathrm{cells} / \mathrm{ml}$ were incubated in triplicate in the presence or absence of various concentrations of itraconazole for $15 \mathrm{~min}$ at $37^{\circ} \mathrm{C}$. Cells were washed with PBS and resuspended with $5 \mu \mathrm{M}$ DiOC5 for $2 \mathrm{~min}$. Cells were analyzed using FACScan. Data are expressed as mean fluorescence channel (MFC) numbers. If the MFC numbers are decreased, the membrane is considered depolarized, whereas, when MFC are increased, it is considered hyperpolarized.

\section{Results}

Effect of itraconazole on intracellular daunorubicin accumulation. Cells were incubated with daunorubicin in the presence or absence of various concentrations of itraconazole and intracel- 
lular accumulation was measured using FACScan. Data in Fig. 1 show significantly lower basal levels of daunorubicin accumulation in P388/ADR cells as compared to P388-sensitive cells. Furthermore, itraconazole, in a dose-dependent manner, restored the daunorubicin accumulation in P388/ADR to a level comparable to that of $\mathrm{P} 388$ cells.

Effect of itraconazole on drug sensitivity. Drug sensitivity was measured, using inhibition of ${ }^{3} \mathrm{H}$ thymidine incorporation as an indicator. Cells were incubated in the presence or absence of various concentrations of itraconazole and pulsed with ${ }^{3} \mathrm{H}$ thymidine. Data are expressed as concentrations of daunorubicin that inhibited ${ }^{3} \mathrm{H}$ thymidine incorporation by $50 \%$ (IC/50). Data in Table I show that itraconazole, in a dose-dependent manner, reversed drug resistance in MDR P388/ADR cells to a level comparable to P388 cells.

Itraconazole corrects altered plasma membrane potentials in P388/ADR. Cells were incubated in the presence or absence of various concentrations of itraconazole and plasma membrane potentials were measured with DiOC5 dye, using FACScan. Fig. 2 shows that plasma membrane potentials of P388/ADR cells were decreased (plasma membrane depolarized) as compared to P388 cells and itraconazole at a concentration of $0.05 \mu \mathrm{g} / \mathrm{ml}$ corrected the altered plasma membrane potentials in P388/ADR but had no effect on P388 cells.

\section{Discussion}

The development of acquired MDR is one of the major obstacles to the chemotherapeutic cure of cancer. A number of mechanisms have been proposed for MDR (reviewed in references 1-3). Several studies have shown an association between MDR and decreased accumulation and enhanced drug efflux, and over expression of P-glycoprotein, a metabolic active efflux pump $(1,2)$. In addition, increased levels of glutathione, glutathione $\mathrm{S}$ transferase, and toposiomerase II have been documented as mechanisms for MDR (reviewed in reference 3). It is apparent that different mechanisms for MDR are operational in different tumor cell lines. It is also likely that there are mechanisms for MDR yet to be discovered.

Itraconazole is a new orally active triazole antifungal agent (9) which was synthesized in 1980 and demonstrates a broad

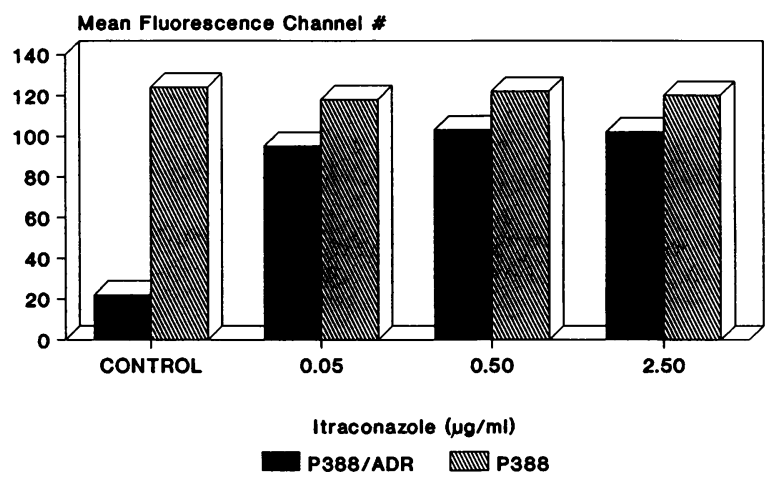

Figure 2. Effect of itraconazole on plasma membrane potentials in P388 and P388/ADR cells. Cells were incubated with DiOC5 dye in the presence or absence of various concentrations of itraconazole and plasma membrane potentials were measured, using FACScan. Data are expressed as mean fluorescence channel numbers. Decreased channel numbers represent depolarization, whereas increased channel numbers represent hyperpolarization. All experiments were done in triplicate and at least three separate experiments were done.

spectrum of activity against a number of fungal species (12, 13). Sterol biosynthesis inhibition at the stage of C-14 demethylation is the primary mode of action (8). Recently, a synergism between triazole agents ketaconazol, itraconazole, and cyclosporin A (CsA) has been reported $(10,14)$. We have shown earlier that CsA reverses multidrug resistance in tumor cells both in vitro and in vivo $(5,6)$. Furthermore, we showed that CsA also corrects altered plasma membrane potentials in MDR tumor cells (11). Therefore, we examined the effect of itraconazole on intracellular drug accumulation, drug sensitivity, and plasma membrane potentials in murine P388/ADR cells. Our study shows that itraconazole, in a dose-dependent manner, corrected intracellular daunorubicin accumulation and reversed the drug resistance to daunorubicin. We also observed some direct antitumor effect of itraconazole, as assessed by an inhibition of DNA synthesis by P388/ADR cells (data not shown). In addition, itraconazole corrected altered plasma membrane potentials in P388/ADR cells. The latter effect of itraconazole was not unexpected because itraconazole has been

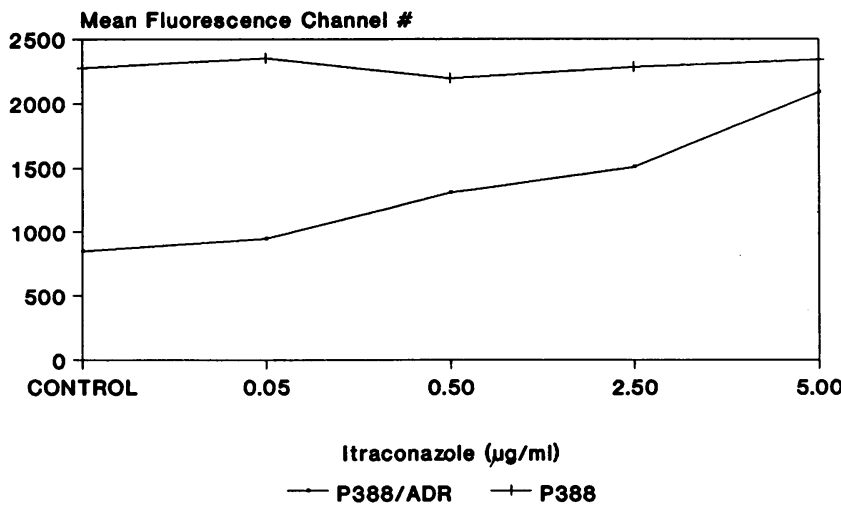

Figure 1. Effect of itraconazole on DNR accumulation. P388 and P388/ADR cells were loaded with $4 \mu \mathrm{M}$ daunorubicin in the presence or absence of various concentrations of itraconazole for $45 \mathrm{~min}$ and DNR uptake is measured, using FACScan. Data are expressed as mean fluorescence channel numbers.
Table I. Effect of Itraconazole on IC/50 of P388/ADR to Daunorubicin

\begin{tabular}{lcc}
\hline Cell line & Itraconazole & Daunorubicin \\
\hline & $\mu g / m l$ & $M$ \\
P388 & None & $1.4 \times 10^{-6}$ \\
P388/ADR & None & $7.2 \times 10^{-6}$ \\
P388/ADR & 0.05 & $3.8 \times 10^{-6}$ \\
P388/ADR & 0.50 & $2.0 \times 10^{-6}$ \\
P388/ADR & 2.50 & $1.3 \times 10^{-6}$
\end{tabular}

$1 \times 10^{6} \mathrm{cells} / \mathrm{ml}$ were incubated with various concentrations of daunorubicin in the absence or presence of three different concentrations of itraconazole, at $37^{\circ} \mathrm{C}$ for $4 \mathrm{~h}$. Cells are pulsed with ${ }^{3} \mathrm{H}$ thymidine, and ${ }^{3} \mathrm{H}$ thymidine incorporation was measured using scintillation counter. IC/50 represents the concentration of daunorubicin to inhibit DNA synthesis by $50 \%$. 
shown to interfere with the plasma membranes of parasitic forms of fungi (8). The concentrations of itraconazole that reversed MDR were well within the range of plasma levels observed in normal volunteers (15) or in patients with fungal infections treated with therapeutic dosage of itraconazole (16). In this study we observed a lack of correlation between the concentrations of itraconazole that corrected all the three parameters studied. Ganapathi et al. (17), and Ganapathi and Grabowski (18) also observed a lack of correlation between the concentrations of trifluoperazine-induced reversal of drug resistance and its effect on doxorubicin accumulation. Furthermore, we have also observed that the concentration of cyclosporin A that corrected the altered plasma membrane potential in L100 acute lymphatic leukemia cell line (11) was much lower than that resulted in the reversal of MDR (6). Although the mechanism(s) of itraconazole-induced reversal of daunorubicin resistance is not known, it is likely that multiple mechanisms are involved including cytochrome $\mathrm{P}-450$ and P-glycoprotein. In any event, itraconazole is a potential candidate for clinical use in human for the reversal of MDR in malignancies. It has an advantage over CsA and other agents in that itraconazole has no adverse effect on significant cardiovascular or endocrine systems and is not immunosuppressive (cited in reference 16). Furthermore, it has an added advantage because of its antifungal activity because patients with malignancies, especially those treated with chemotherapeutic agents, are at increased risk for fungal infections.

\section{References}

1. Bradley, G., P. F. Juranka, and V. Ling. 1988. Mechanisms of multidrug resistance. Biochim. Biophys. Acta. 948:87-128.

2. Gottesman, M. M., and I. Pastan. 1988. Resistance to multiple chemotherapeutic agents in human cancer cells. Trends Pharmacol. Sci. 9:54-58.

3. Lazo, J. S., and R. R. Bahnson. 1989. Pharmacological modulators of DNA-interactive antitumor durgs. Trends Pharmacol. Sci. 10:369-373.
4. Juranka, P. F., R. L. Zastawny, and V. Ling. 1989. P-glycoprotein: multidrug resistance and a supefamily of membrane associated transport proteins. FASEB (Fed. Am. Soc. Exp. Biol.) J. 3:2583-2592.

5. Slater, L. M., P. Sweet, M. Stupecky, and S. Gupta. 1986. Cyclosporin A corrects daunorubicin resistance in Ehrlich ascites carcinoma. Br. J. Cancer. 54:235-239.

6. Slater, L. M., P. Sweet, M. Stupecky, and S. Gupta. 1986. Cyclosporin A reverses vincristine and daunorubicin resistance in acute lymphatic leukemia in acute lymphatic leukemia in vitro. J. Clin. Invest. 77:1405-1408.

7. Van Cutsem, J. F., F. Van Gerven, M. A. Van de Ven, M. Borger, and P. A. J. Janssen. 1984. Itraconazole, a new triazole that is orally ctive against aspergillosis. Antimicrob. Agents Chemother. 26:527-534

8. Berg, D., M. Plempel, K.-H. Buchel, G. Holmwood, and K. Stroech. 1988. Sterol biosynthesis inhibitors. Secondary effects and enhanced in vitro efficacy. Ann. NY Acad. Sci. 544:338-347.

9. Hay, R. J., B. Dupont, and J. R. Graybill. 1987. First International Symposium on Itraconazole: A summary. Rev. Infect. Dis. 9(Suppl. 1):S1-S3.

10. Trenk, D., W. Brett, E. Jahnchen, and D. Birnbaum. 1987. Time course of cyclosporin A/itraconazole interaction. lancet. ii:1335-1336.

11. Vayuvegula, B., L. M. Slater, J. Meador, and S. Gupta. 1988. Correction of altered plasma membrane potentials: a possible mechanism of cyclosporin A and verapamil reversal of pleiotropic drug resistance in neoplasia. Cancer Chemother. Pharmacol. 22:163-168.

12. Tucker, R. M., P. L. Williams, E. G. Arathoon, and D. A. Stevens. 1988. Treatment of mycoses with itraconazole. Ann. NY Acad. Sci. 544:451-470.

13. Kan, V. L., and J. E. Bennett. 1988. Efficacies of four antifungal agents in experimental murine sporotrichosis. Antimicrob. Agents Chemother. 32:16191623.

14. Dieperink, H., and J. Moller. 1982. Ketoconazole and cyclosporin. Lancet. ii: 1217.

15. Hardin, T. C., J. R. Graybill, R. Fetchick, R. Woestenborghs, M. G. Rinaldi, and J. G. Kuhn. 1988. Pharmacokinetics of itraconazole following oral administration to normal volunteers. Antimicrob. Agents Chemother. 32:1310 1313.

16. Cauwenbergh, G., and J. V. Cutsem. 1988. Role of animal and human pharmacology in antifungal drug design. Ann. NY Acad. Sci. 544:264-269.

17. Ganapathi, R., H. Schmidt, D. Grabowski, M. Melia, and N. Ratliff. 1988. Modulation in vitro and in vivo of cytotoxicity but not cellular levels of doxorubicin by the calmodulin inhibitor trifluoroperazine is dependent on the level of resistance. Br. J. Cancer. 58:335-340.

18. Ganapathi, R., and D. Grabowski. 1988. Differential effect of the calmodulin inhibitor trifluoroperazine in modulating cellular accumulation, retention, and cytotoxicity of doxorubicin in progressively doxorubicin resistant 11210 mouse leukemia cells. Biochem. Pharmacol. 37:185-193. 